\title{
High Value Number
}

National Cancer Institute

\section{Source}

National Cancer Institute. High Value Number. NCI Thesaurus. Code C42853.

Indicates the upper limit permissible for a value. 